\title{
Nanoscale
}

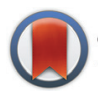

CrossMark

\& click for updates

Cite this: Nanoscale, 2017, 9, 2768

\section{Imaging and structural studies of DNA-protein complexes and membrane ion channels $\dagger$}

\author{
M. Marini,,$^{a}$ T. Limongi, $t^{a}$ A. Falqui, ${ }^{b}$ A. Genovese, ${ }^{b}$ M. Allione, ${ }^{a}$ M. Moretti, ${ }^{a}$ \\ S. Lopatin, ${ }^{\mathrm{C}}$ L. Tirinato, ${ }^{a}$ G. Das, ${ }^{a}$ B. Torre, ${ }^{a}$ A. Giugni, ${ }^{a}$ F. Cesca, ${ }^{d}$ F. Benfenati ${ }^{d}$ and \\ E. Di Fabrizio*a
}

\begin{abstract}
In bio-imaging by electron microscopy, damage of the sample and limited contrast are the two main hurdles for reaching high image quality. We extend a new preparation method based on nanofabrication and super-hydrophobicity to the imaging and structural studies of nucleic acids, nucleic acid-protein complexes (DNA/Rad51 repair protein complex) and neuronal ion channels (gap-junction, $\mathrm{K}^{+}$and $\mathrm{GABA}_{\mathrm{A}}$ channels) as paradigms of biological significance and increasing complexity. The preparation method is based on the liquid phase and is compatible with physiological conditions. Only in the very last stage, samples are dried for TEM analysis. Conventional TEM and high-resolution TEM (HRTEM) were used to achieve a resolution of 3.3 and $1.5 \AA$, respectively. The EM dataset quality allows the determination of relevant structural and metrological information on the DNA structure, DNA-protein interactions and ion channels, allowing the identification of specific macromolecules and their structure.
\end{abstract}

Received 10th October 2016, Accepted 16th January 2017

DOI: 10.1039/c6nr07958j

rsc.li/nanoscale ization of various types of complex biological systems isolated under true physiological conditions, without the need to crystallize or freeze them, and by reducing the damage of the electron beam, ${ }^{2}$ can potentially play a key role in understanding the regulation of important processes in physiology and pathology. The possibility of mapping DNA-protein or proteinprotein interactions at $\AA$ resolution and directly studying the structure of single ion channels in their native environment will make it possible to elucidate gene expression and signal transduction mechanisms. Although the electrophysiology of membranes is very well understood and several experimental and theoretical methods have been developed, ${ }^{3}$ a detailed characterization of the cell membrane through high-resolution imaging is still missing. ${ }^{4}$ Moreover, cell membranes with their associated proteins are the targets for several extracellular and intracellular molecules as well as for drugs. The possibility to study how the plasma membrane and the cytoskeleton respond to several pathological stresses and how their composition affects signaling and cell responses to physical-chemical stimuli and drugs will foster the establishment of novel therapeutic approaches, such as membrane-lipid therapy, aimed at developing strategies for the modulation of membrane-lipid composition. ${ }^{5}$

\section{Results and discussion}

The present approach can be considered a unifying technique that can be applied to different biological systems where the 


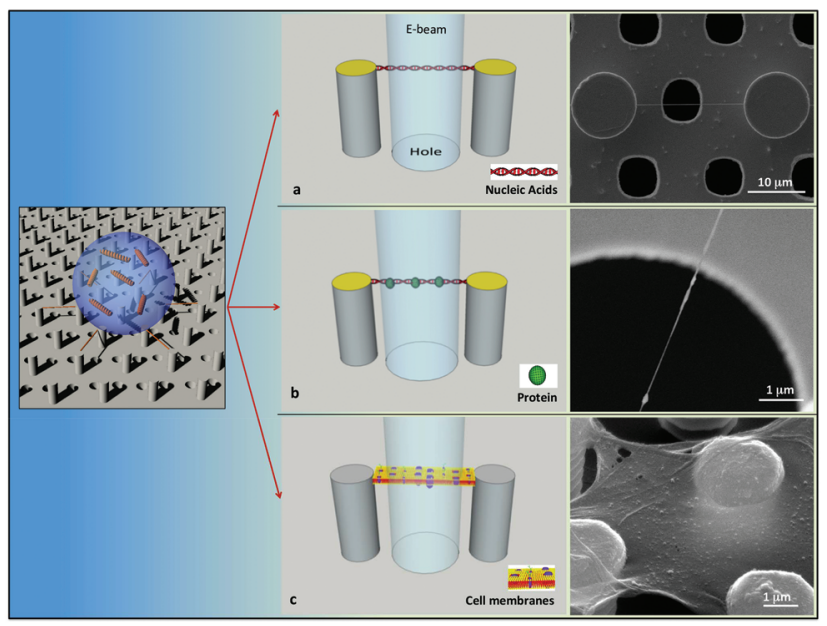

Fig. 1 General scheme and results of the evaporation process. On the left, the deposition of a droplet containing the sample of interest on a super-hydrophobic micro-patterned device is shown. (a, c) After the subsequent evaporation step, the three samples ( $a, D N A ; b$, DNAprotein complex; $c$, plasma membrane patch) are suspended between adjacent $\mu$-pillars. On the right, the respective SEM pictures are reported.

super-hydrophobic device is the universal substrate that allows the elimination of the parasitic electron scattering background. This strongly affects the improvement of TEM contrast image of biological samples without the help of any staining techniques. This approach is depicted in Fig. 1. A droplet containing the sample of interest is deposited onto a super-hydrophobic substrate. We studied 3 cases. After the droplet evaporation, in (a) a suspended DNA fiber is ready to be imaged by TEM, in (b) the DNA/Rad51 complex is suspended as in (a), and in (c) a mouse neuronal plasma membrane is suspended from a proper solution. In the third column of Fig. 1, SEM images are reported for each of the three systems, ready to be imaged by TEM.

New super-hydrophobic devices, shown in ESI Fig. S1, $\dagger$ were designed and engineered to improve the present techno- logy used in this study. The super-hydrophobic device (ESI Fig. S1†) was further optimized by controlling the thickness of a hydrophobic capping layer deposited on silicon pillars to tune the hydrophobicity and maximize the contact angle (about $170^{\circ}$ ), thus obtaining an optimal number of DNA bundles with controlled diameter. Details of these procedures are reported in the Experimental section.

\section{Direct imaging of DNA filaments}

This case was studied in two previous papers ${ }^{1,6}$ where several characteristic lengths of the imaged double strand (ds) DNA were directly measured for the first time. Here, we report in Fig. 2 additional images showing an improvement of the image contrast and resolution. In Fig. 2a, a low magnification TEM image of a suspended dsDNA bundle displays a narrow region with a diameter below $50 \AA$ A. Further magnified HRTEM images, Fig. $2 \mathrm{~b}$ and $\mathrm{c}$ with enhanced contrast, show clearly the backbone and the inner structure of dsDNA. The inter-base distance of approximately $2.6 \AA$ was determined; the experimental nucleotide's lengths were directly measured by the contrast curve and the backbone (phosphate + sugar, 5.3 $\AA$ ) was clearly distinguished from the pyrimidine base, $3.8 \AA$ as in Fig. 2d.

The beam energy was set at $80 \mathrm{keV}$ and the typical dose was about 200 electrons per Ångstrom ${ }^{2}$. Additional details are given in the Experimental section.

\section{Direct imaging of the DNA/Rad51 complex}

SSDNA from calf thymus of approximately $50 \mathrm{~kb}$ was evaluated for this analysis. A comparative SEM imaging was performed both on ssDNA and ssDNA/Rad51 complexes and is reported in Fig. 3. In Fig. 3a and b ssDNA of different diameters, ranging from approximately 20 to $7.5 \mathrm{~nm}$, are suspended and stretched between adjacent $\mu$-pillars. After ssDNA incubation with the protein Rad51, clusters randomly distributed along the stretched DNA filament could be clearly seen as in Fig. 3c and $\mathrm{d}$. These aggregates are attributable to the presence of Rad51 bound to the ssDNA filament.
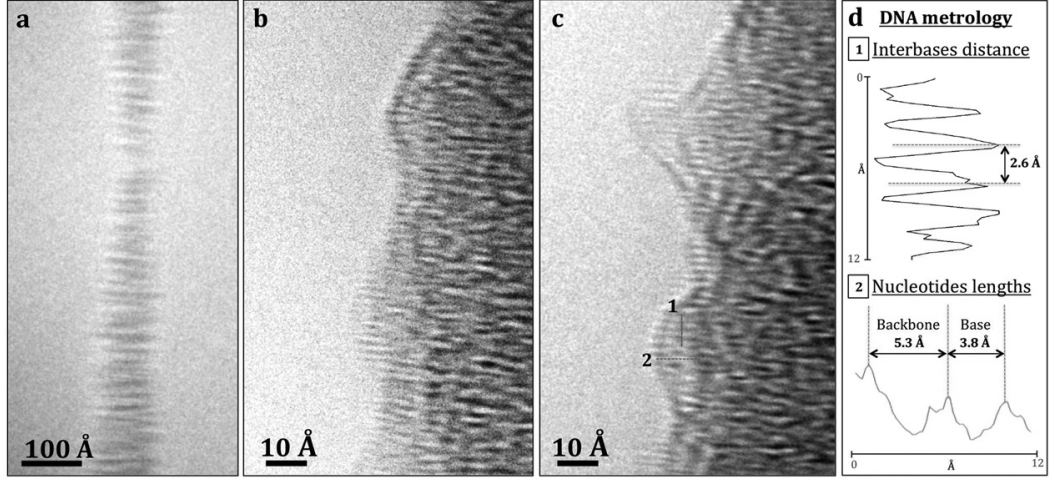

Fig. 2 DNA HRTEM images. (a) Low magnification image of a DNA bundle. (b, c) Higher magnification images with fine structural details and (d) detailed measurements of the inter-base distances, backbone and base lengths. 


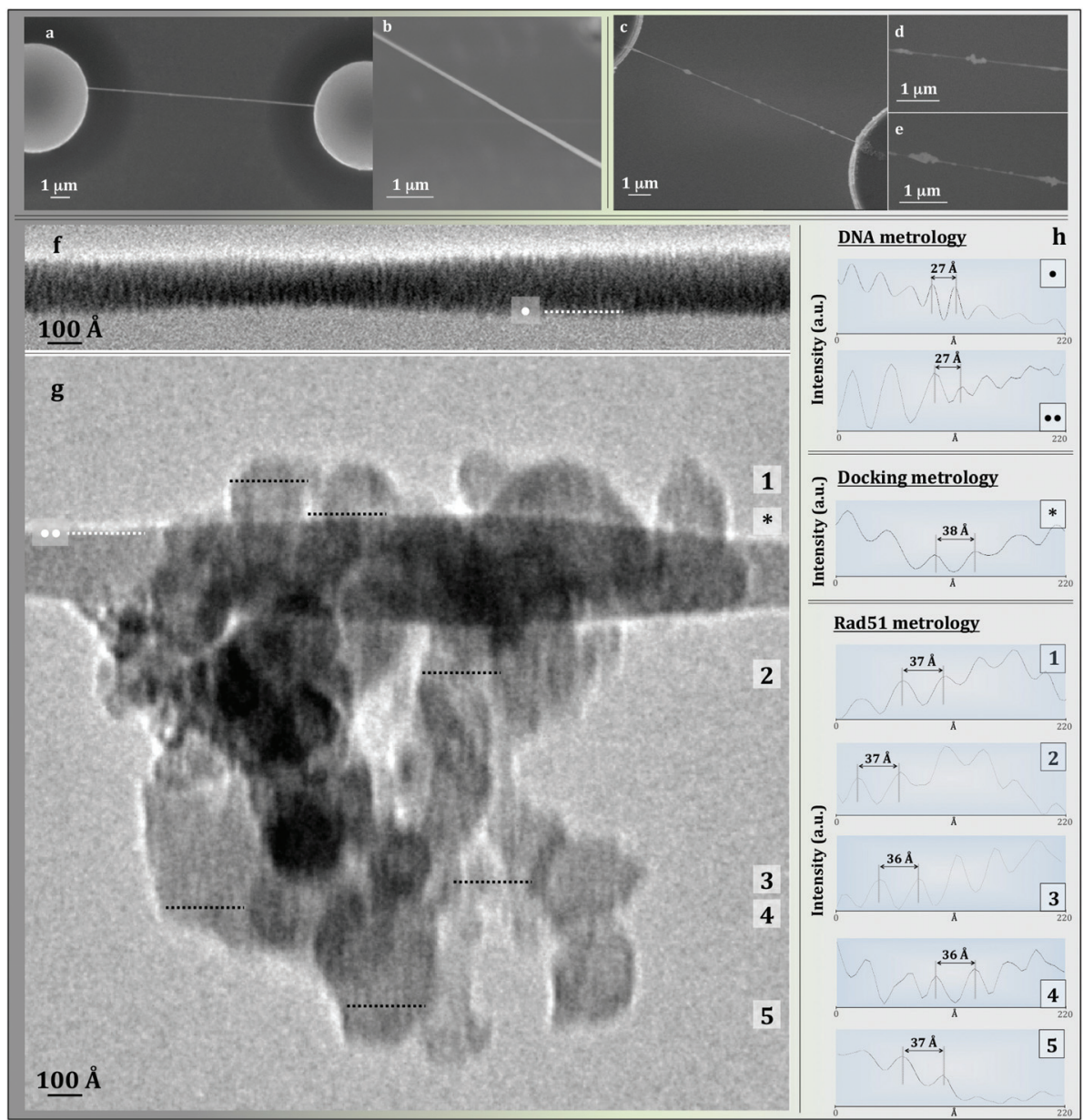

Fig. 3 SEM and TEM images with metrology of the sSDNA/Rad51 complex. DNA molecules deposited on super-hydrophobic devices, resulting in well tensed suspended fiber between $\mu$-pillars. (a) Low magnification top view image of ssDNA after droplet evaporation and (b) higher magnification image of the DNA filament suspended between two $\mu$-pillars. (c-e) SEM images after deposition of the DNA/Rad51 complex on silicon $\mu$-pillars at low (c) and high (d, e) magnification. Clusters of DNA-protein complexes are clearly visible. (f) DNA bundle of $27 \AA ̊$ periodicity. (g) Rad51 complex docked on an ssDNA bundle. Both images were taken with an electron acceleration voltage of $100 \mathrm{keV}$. In both cases, it is possible to distinguish the DNA periodicity of $27 \AA(h)$, in good agreement with the previously reported values. ${ }^{1,6}$ The periodicity measured in the presence of the DNA/Rad51 complex (h, insets), ranging from 36 to $38 \AA$, is attributable to the Rad51 repair protein, suggesting a bi-heptameric conformation. ${ }^{12}$

The suspended ssDNA/Rad51 complex bundles and their portion aligned to the substrate holes of the array were then used for direct imaging by TEM (further SEM images are reported in ESI Fig. S2 $\dagger$ ). Structural analysis by TEM is reported in Fig. 3. The comparison of DNA and the ssDNA/ Rad51 complex is reported in Fig. $3 a$ and $b$, respectively. In Fig. 3f-h suspended DNA/Rad51 is reported with details of the docking region and those of the protein-protein ordered cluster, as evidenced by the regular contrast intensity modulation pattern in the image. In Fig. 3f, the TEM image of the sSDNA bundle is reported as a metrological reference for the suspended DNA/Rad51 complex as in Fig. 3g. In addition to the DNA periodicity of $27 \AA$ detectable in both cases (out of the docking region), details of the ssDNA/Rad51 docking region and of the protein-protein ordered aggregate were observed, as evidenced by the regular vertical intensity modulation pattern along the entire protein as clearly measurable in Fig. 3h (a sketch of the complex is reported in ESI Fig. S3†). We analyzed in detail the docking region of Rad51 on ssDNA and proteinprotein long range order. The full construct of ssDNA/Rad51 shows the protein docking period and how this periodicity is conserved throughout the protein cluster, as measured in the locations in the insets of the picture. The TEM intensity modulation profiles in Fig. 3h clearly show the periodicity of the Rad51 in different positions along the constructs. In particular, we can distinguish the docking period of Rad51 on ssDNA, which measures about $38 \AA$, and the DNA period of $27 \AA$. We note that the $\operatorname{Rad} 51$ periodicity is maintained for a long distance, up to $150 \mathrm{~nm}$ far from the DNA bundle. This periodicity is led by the protein docking on DNA and is compatible with a close packing of Rad51 subunits via protein-protein interactions (see below). Several cellular DNA-binding proteins exist as ring-shaped homo-oligomers and modulate their biological activity by assembly and disassembly. ${ }^{7,8}$ 
Proteins of the Rad51 family can spontaneously self-associate into heptameric rings ${ }^{9-13}$ and change their conformation upon ATP binding. This event allows the formation of the active DNA-bound filamentous form of Rad51, suitable for the recognition of breaks along the nucleic acid filament and the consequent strand displacement. ${ }^{14-16}$ As previously reported, the switch between the two conformations is driven by several environmental conditions such as divalent cation and protein concentration or ATP presence. ${ }^{17,18}$ Moreover, the ring-shaped heptamers have been observed only in the absence of nucleic acids in solution. ${ }^{14,19}$

Starting from the Rad51 structure, we propose a model in Fig. 4 where two heptameric Rad51 proteins are packed, vertically with respect to their equatorial plane as in Fig. 4a. The packing period corresponds to the docking period with DNA and is $38 \AA$, as in Fig. 4b and consistent with that calculated from the TEM simulation in Fig. 4c.

In our model, the docking period can be explained as the $\beta$-sheet binding recognition of DNA groves as in Fig. $4 \mathrm{~d}$ and e, according to an accepted hypothetical mechanism. Herein we demonstrate that the Rad51 heptameric ring-shaped form is stable also in the presence of ssDNA, suggesting that a preliminary recognition event between the two molecules occurs before the nucleoprotein formation. The interaction between several heptameric Rad51 structures occurs mostly by polar side-chain interactions ${ }^{14}$ and originates the ordered longrange periodic structures. ${ }^{18}$

Nucleoproteins show non-specific interactions between the side chains of proteins with a positive charge and the negative charge of DNA strands with topological dependent affinity. ${ }^{20}$ We hypothesize that electrostatic and hydrophobic interactions are responsible for the non-sequence-specific Rad51 docking on ssDNA. Stacking interactions may involve the intercalation of aromatic amino acids (tyrosine, tryptophan, phenylalanine) between the rings of the DNA bases. Positively charged amino acids (such as lysine, arginine and histidine) can interact by hydrogen bonds with the phosphate and deoxyribose groups of the DNA backbone; in addition, the conformation of the

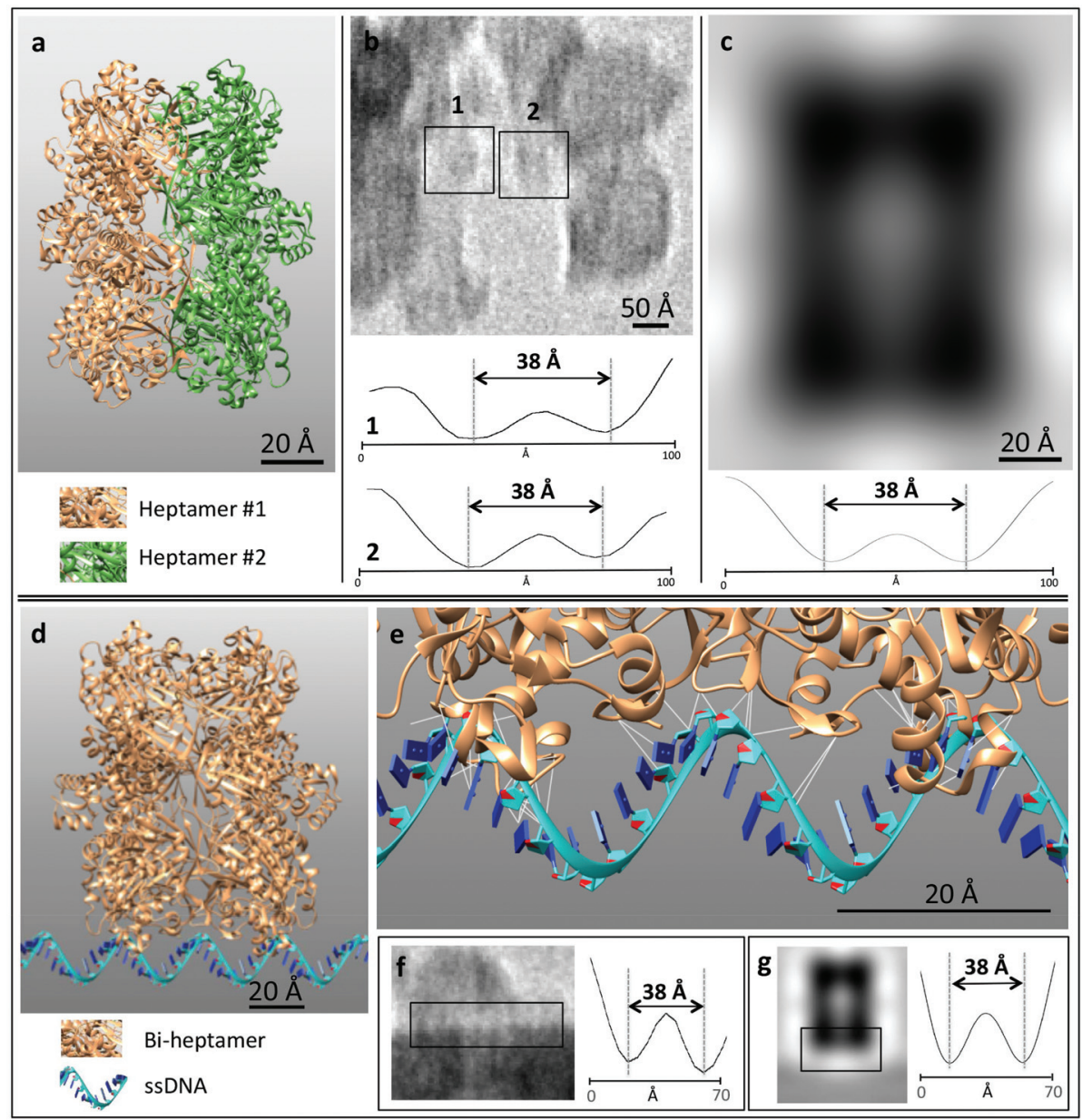

Fig. 4 Rad51 bi-heptamers and docking on DNA. (a) Two heptameric Rad51 proteins (b) directly imaged by TEM, showing a periodicity of $38 \AA$, coherently with the profile obtained by the simulation (c). (d) Docking model of two heptameric Rad51 proteins on ssDNA, due to aspecific electrostatic interactions (e). The docking periodicity of $38 \AA$ is directly measurable (f). The value obtained is in good agreement with the periodicity of the simulated ssDNA/Rad51 docking $(\mathrm{g})$. The simulated defocus in panels $\mathrm{c}, \mathrm{g}$ is $-1.5 \mu \mathrm{m}$. 
grooves provides suitable surface charges for non-covalent bonding. In Fig. 4e DNA and Rad51 bonds are explicitly indicated. In Fig. 4f reported a detail of the docking area and the period measured by the contrast curve obtained by the experimental image. In Fig. $4 \mathrm{~g}$ we report the image simulation, limited to 2 Rad51 heptameric molecules over the DNA bundle, where the docking periodicity of $38 \AA$ is well reproduced (see ESI Fig. $\mathrm{S} 4 \uparrow$ for further details). We note that, to our knowledge, this is the first experimental evidence of this binding-recognition mechanism. We argue that the docking/ recognition mechanism described above is at the origin of the repair mechanism of Rad51 due to its dependence on local topography and strand displacement. A multiscale molecular dynamics would be necessary to clarify these aspects.

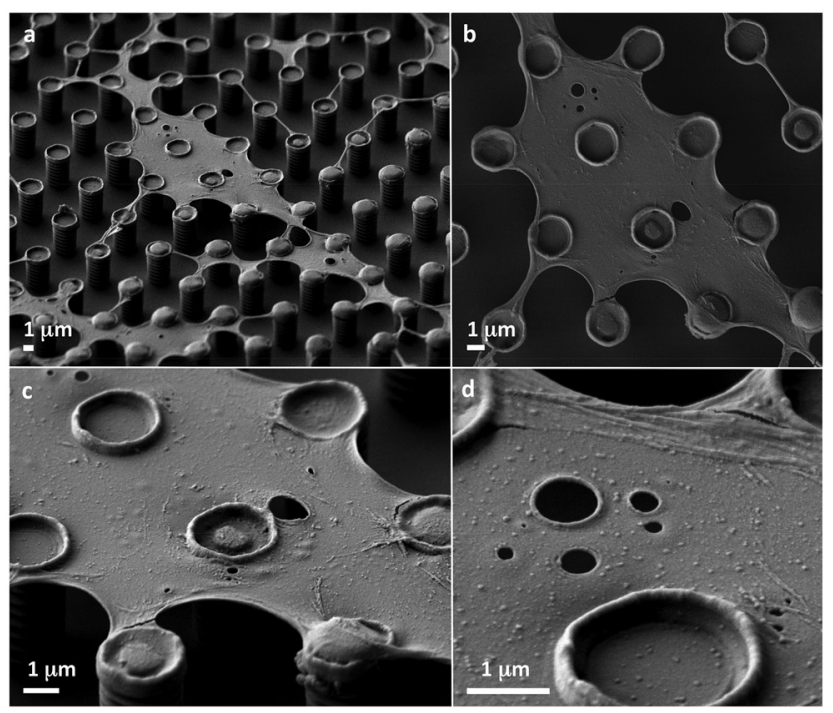

Fig. 5 SEM images of cortical neuronal membranes suspended over the SHS. (a) Low-magnification view of parts of the suspended membranes, with the substrate tilting angle of 35 degrees. (b) Top view (untilted sample) of the membrane around the central location of panel a. (c) 35 degrees tilting view at higher magnification of the same location shown in panel b. (d) 35 degrees tilting high-magnification image showing aggregates of membrane proteins.

\section{Direct imaging of native neuronal membranes}

In the case of membranes, specimen preparation is undoubtedly the most crucial and delicate step for obtaining highresolution images to avoid sample damage, poor contrast and artifacts, such as dehydration or morphology alteration when embedding in hard resin and metallization procedures. Here, we demonstrate the application of micro-fabricated SHS to suspensions of neuronal membranes to achieve substrate-free imaging. The detailed preparation process for the membrane suspension and drying is reported in the Experimental section.

\section{Analysis of membrane samples by SEM imaging}

After the application of the membrane suspension, scanning electron microscopy images of the devices showed large patches where the membranes were clearly suspended on the pillars of the SHS device. Low magnification SEM images (Fig. 5a and b) confirmed that isolated membranes were well tensed in a sort of planar layer. High magnification SEM images (Fig. 5c and d) showed surface details where the structures on the nanometer length scale are membrane proteins. These images are reminiscent of those obtained by means of freeze fracture electron microscopy, which allows the examination of the oligomeric structure and molecular asymmetry of integral plasma membrane proteins. $^{21}$

\section{Analysis of membrane samples by TEM}

The sample preparation for TEM is usually more laborious and delicate than that needed for SEM imaging, as it usually includes additional steps such as sample resin embedding, ultrathin sectioning and staining of thin and ultrathin sections. However, by using SHS devices, the preparation becomes much simpler and less aggressive to the integrity of the membrane structure, there being necessary only a fixing step with $2 \%$ glutaraldehyde.

The suspended and stretched membrane portion corresponding to the substrate holes is shown at low magnification in Fig. 6a. The membrane electron transmission was directly deducible from the TEM image by measuring the contrast
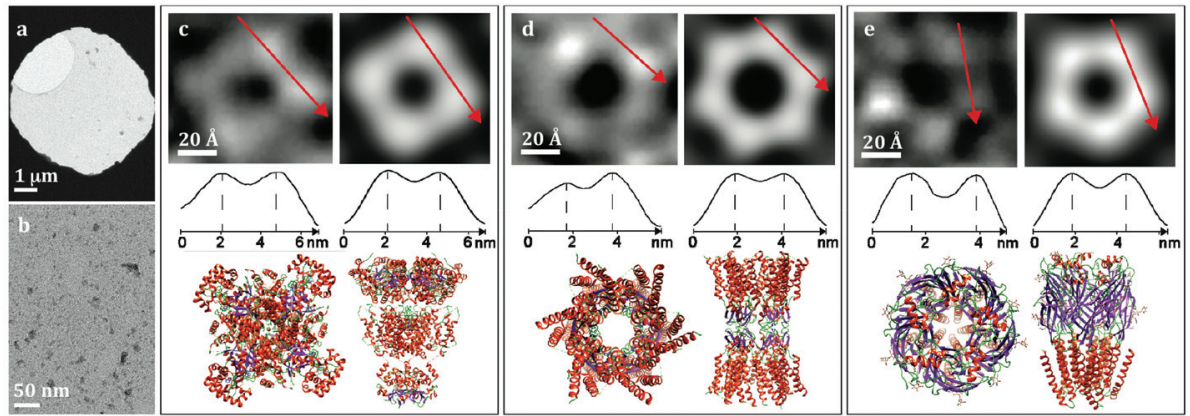

Fig. 6 TEM images of channels and receptors. Survey TEM imaging at very low (a) and intermediate (b) magnification of the suspended protein membrane. (c-e) Sequence of TEM image details, TEM image simulations, corresponding contrast profiles, and plots of structural models used for the simulations: K-channel ${ }^{22}$ (PDB file: 3LNM) (c), Ca Gap Junction ${ }^{23}$ (PDB file: 5ERA, d), and GABA ${ }^{24}$ (PDB file: 4COF, e). The simulated defocus in $\mathrm{c}-\mathrm{e}$ is $1 \mu \mathrm{m}$. 
between the membrane and the hole. We measured a transmissivity of $96 \%$, compatible with Monte Carlo simulation transmittance for a $100 \mathrm{keV}$ electron beam of a single lipid bilayer, indicating that the suspension process does not induce any membrane folding. At higher magnification in Fig. 6b, numerous aggregates were observed on the membrane surface distributed as single particles or in small clusters, likely representing membrane proteins and protein complexes. Their distribution on the membrane plane represents the twodimensional projection of the structures observed in high resolution SEM (Fig. 5d).

By further increasing the magnification as in Fig. 6c, a number of pore structures of different symmetries, sizes and topographies clearly appeared. After an analysis with the PDB database, we could distinguish 3 types of pores that could be assigned to ion channels as reported in Fig. 6c-e. The corresponding assignment of the pore structures to specific ion channels, namely $\mathrm{K}$ channel, $\mathrm{Ca}$ gap junction and $\mathrm{GABA}_{\mathrm{A}}$ receptor channels, ${ }^{22-24}$ was accomplished after TEM image simulations on which the experimental conditions (a single suspended membrane layer (no substrate), $100 \mathrm{keV}$ beam energy, $3 \AA$ resolution, $1 \mu \mathrm{m}$ defocus) were imposed. The TEM simulation clearly agrees with the high-contrast images of the experimental samples.

In Fig. 6, panels c-e, by using the experimental image contrast curve we could determine the characteristic channel lengths of each ion channel at sub-nm accuracy, together with the axial rotation symmetry group of each pore that gave: $\mathrm{C}_{4}$ for $\mathrm{K}$ channel, $\mathrm{C}_{6}$ for Ca gap junction and $\mathrm{C}_{5}$ for $\mathrm{GABA}_{\mathrm{A}}$ receptor. Due to the strong agreement between the experimental measurements and the simulation, based on the PDB structure we are fully confident of the correct assignments proposed in Fig. 6 .

Another aspect that is worth mentioning at this stage is the information content in terms of structures in the suspended area accessible by TEM. Each hole in the SHS device allows access to a membrane area of about $20 \mu \mathrm{m}^{2}$. Through image recognition software, we filtered the channel-like topography in our TEM images and found an overall density of about 3000 pores per $\mu \mathrm{m}^{2}$. These data are compatible with data found in the literature ${ }^{25-27}$ in which, for instance, a density of about 500 $\mathrm{GABA}_{\mathrm{A}}$ receptor channels per $\mu \mathrm{m}^{-2}$ of neuronal membrane was determined by fluidics measurements. A map of the pore-structure localization is reported in Fig. 6. Considering the whole area per hole that is accessible in a TEM image, we can estimate that the number of accessible pores is in the range of 60000 . This amount of information is comparable to the "single particle" statistical sample needed in cryo-EM. ${ }^{28,29}$ In other words, the present method lends to further use in single particle structural determination, with the evident advantage that the membrane under study is a real native biological system.

In Fig. 7 we report the structure recognition procedure. In Fig. 7a, the characteristic structures, whose shape is amenable to ion channel receptors and to multimeric proteins, have been placed in evidence on the direct TEM image of the neuronal plasma membrane. In the bottom panels of Fig. 7, the segmentation process for selecting objects of interest (different

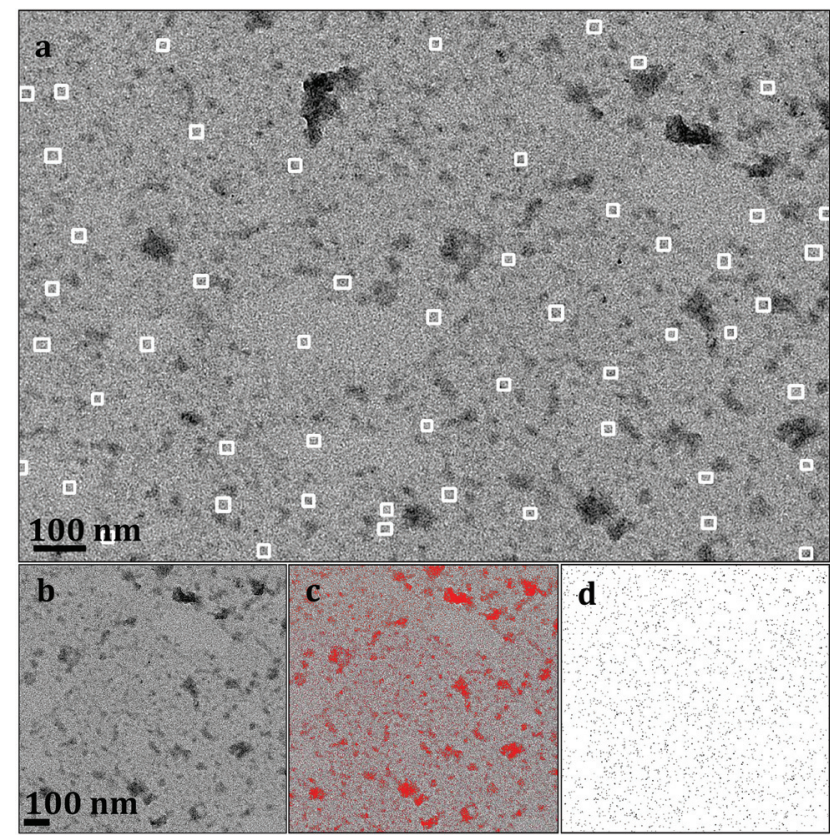

Fig. 7 TEM image of cortical neuron cell membranes and image particle analysis. (a) Some representative channel receptors are in evidence (white boxes) as the region of interest. (b) A zone of one square micrometer of panel (a) is selected. (c) Threshold image applied. (d) Resultant structures isolated and counted.

kinds of ion channels) from other objects (artifacts, fragments or clusters of pieces of broken membranes) has been shown in order to show the counting procedure. Selecting an area of $1 \mu \mathrm{m}^{2}$ from the whole image (Fig. $7 \mathrm{~b}$ ), a segmented image based on threshold intensity has been separated from the uninteresting structures (Fig. 7c). Then only particles with sizes ranging from 5 to 50 pixel units within a $0.6-1$ range of circularity have been selected (Fig. 7c) and counted. This resulted that in the selected region of interest about 2800 channel receptors were present.

\section{Lipid bilayer imaging}

As a further imaging achievement, high-resolution TEM images at the membrane edges showed local membrane folding, likely due to the stretching force related to the superhydrophobic suspension mechanism as in Fig. 8a and b. In those cases, we often found that, in this limited location, the membrane folded over itself. This configuration of the sample allowed us to resolve and measure, in a cross-section of the membrane, the lipid bilayer of each fold of the membrane. In Fig. $8 \mathrm{~b}$ we depicted the "edge folding" of the membrane, to better interpret the TEM images. In fact, the edge folding configuration shows the lipid bilayer two times with an interspaced amorphous layer of a saline residue after the drying step. In Fig. 8c and d the length of each lipid bilayer is accurately measured through the contrast curve giving a length of $50 \AA$, in good agreement with what is known from experience. This quantitative length measurement is possible thanks to the high contrast image originating from the lack of any back- 


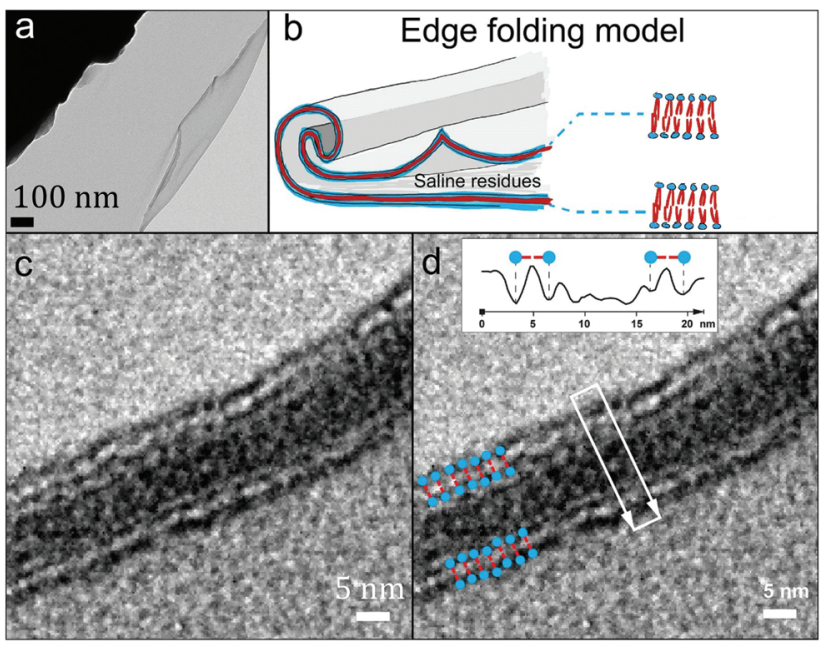

Fig. 8 Membrane cross-section analysis. The TEM cross-section of a membrane that appears broken and partially folded on itself is reported in (a). In the art style draft (b), folding is further schematized: the phospholipid bilayer is highlighted, and the polar head region and the hydrophobic tail region are represented in blue and in red respectively. In (c) and (d), high-magnification TEM cross-section image and metrology of the neuronal membrane bilayers from the contrast curve are reported.

ground scattering. We note that edge folding suggests a future development in the technique to further include cross section imaging of ion channels. In fact, there are several ways to make a controlled cut on the suspended membrane. We refer to the AFM scratch technique or ion beam milling or femtosecond laser ablation that could be used for this application in order to gain 3D topography information in the imaging of proteins and ion channels embedded in the lipid bilayer.

It is far beyond the scope of this paper the analysis of the whole membrane content, but the great deal of information in the TEM image will be used to further assign the temporarily unknown structures isolated by the method described above.

\section{Experimental}

\section{Super-hydrophobic device realization}

For the realization of these samples, $50 \mu \mathrm{m}$ thick $\mathrm{Si}<100>4$ " commercial wafers (SiMat, Germany) were used. The pillars and the holes through the sample were defined with two steps of optical lithography. In both cases a positive resist was used (AZ 5214, used in positive tone) spun at $3000 \mathrm{rpm}$, giving a final thickness of approximately $2 \mu \mathrm{m}$, with an exposure dose of $80 \mathrm{~mJ} \mathrm{~cm} \mathrm{~cm}^{-2}$. Both lithographic steps were done on the same side of the wafer. The first lithography defined the pattern of holes where the pillars would stand after the final reactive ion etching step. A consecutive deposition of three metals was made by sputtering titanium $(10 \mathrm{~nm})$, gold $(50 \mathrm{~nm})$ and chromium $(50 \mathrm{~nm})$ in this order. The purpose of the top $\mathrm{Cr}$ layer was to protect the underlying gold layer during dry etching. The Ti layer promotes the adhesion of the upper gold layer on the silicon substrate. The metal deposited on the resist was removed by lift-off in acetone, while sonicating.

Subsequently, a second lithography was re-aligned on top of the previous one, and the resist itself was used as a mask in this second case. Both lithographic steps were performed on a commercial contact aligner machine (EV1000, EV Group Inc., Switzerland) in hard contact mode.

The structures were then etched into the substrate with Deep Reactive Ion Etching (DRIE) of silicon using the so-called Bosch process in a commercial DRIE plasma etching machine (Oxford Instruments, UK). For the etching of the holes through the wafer, $12 \mathrm{~s}$ cycles were used with a $5 \mathrm{~s}$ deposition step using $100 \mathrm{sccm}$ of $\mathrm{C}_{4} \mathrm{~F}_{8}$ as a precursor for the protective polymer (a radiofrequency power (RF) of $5 \mathrm{~W}$ and an inductively coupled plasma (ICP) power of $1300 \mathrm{~W}$ ), and 7 seconds of etching steps, using $\mathrm{SF}_{6}$ as etching reagent $(120 \mathrm{sccm}$, an RF power of $40 \mathrm{~W}$ and an ICP power of $1300 \mathrm{~W}$ ). All of the process was performed with the sample on a stage cooled at $-20{ }^{\circ} \mathrm{C}$. The etching speed was $0.2 \mu \mathrm{m}$ per cycle, thus requiring 250 steps to open holes through the wafer.

After this process, the eventual residual of the resist of the second lithography was removed in an oxygen plasma, and a second DRIE process was performed to create the pillars, using the previously deposited metal as a mask. The parameters used for the second DRIE were the same as those of the first one, apart from the flux of $\mathrm{SF}_{6}$ in the etching step of each cycle, increased to $140 \mathrm{sccm}$ to compensate for the larger area etched in this case. In this process 50 cycles of deposition/etching were used, creating $10 \mu \mathrm{m}$ high pillars. Finally, the top protecting Cr layer on top of pillars was removed with a wet chemical etchant, selectively attaching only chromium and not gold (Chrome Etch Standard, Sigma Aldrich), thus exposing the flat gold surface on the top of each pillar.

The sample prepared in this way was finally coated with a thin layer of hydrophobic polymer, to give a super-hydrophobic behavior to the surface: the deposition step used in DRIE cycles was repeated here, in a single step of 12 seconds, finally leading to a final deposition of a teflon-like fluorocarbon polymer, whose thickness, measured by atomic force microscopy, turned out to be approximately $2 \mathrm{~nm}$.

\section{dsDNA preparation, suspension and imaging}

A detailed study was conducted on the solution composition, including salinity, $\mathrm{pH}$, evaporation and temperature control, to achieve the optimal working conditions. In this work, Lambda dsDNA (NEB) was diluted at a concentration of $50 \mathrm{ng} \mu^{-1}$ in saline buffer ${ }^{30}$ (final concentration: $6.5 \mathrm{mM} \mathrm{NaCl}, 10 \mathrm{mM}$ Tris$\mathrm{HCl} \mathrm{pH} \mathrm{9.3).} \mathrm{The} \mathrm{DNA} \mathrm{working} \mathrm{solution} \mathrm{was} \mathrm{preheated} \mathrm{at}$ $65{ }^{\circ} \mathrm{C}$ for 10 minutes, cooled at $30{ }^{\circ} \mathrm{C}$ for 20 minutes and immediately used. A $5 \mu \mathrm{l}$ droplet was then deposited on the super-hydrophobic device. The silicon substrate used was preheated for 10 minutes prior to use at $25{ }^{\circ} \mathrm{C}$ and kept at this temperature until the end of the evaporation process, occurring at a room temperature of $21{ }^{\circ} \mathrm{C}$. The HRTEM images of DNA bundles were acquired at $80 \mathrm{keV}$ using a Titan 60-300 TEM provided with a high-brilliance field emission electron 
gun (X-FEG), a monochromator (Wien type), a CS (spherical aberration) corrector for the objective lens, and a Gatan Tridiem 865 image filter (GIF). The typical electron dose setting used for HRTEM imaging was about 10-20 electrons per Ångstrom ${ }^{2}$ per second. Imaging was performed by tuning the image corrector and setting the third order Cs parameter to a negative value $(-12 \mu \mathrm{m})$ in order to offset the unbalanced value of the fifth order Cs and enhance the electron contrast of the biological materials (weak phase objects). To increase the signal/noise ratio, HRTEM images were acquired by filtering the zero-loss signal - cutting out the inelastically scattered electrons - with a GIF Tridiem filter and using an exposure time of the CCD camera ranging from 0.3 to $1 \mathrm{~s}$.

\section{Preparation, suspension and imaging of the ssDNA/Rad51 repair protein complex}

SSDNA of calf thymus (Sigma Aldrich) was preheated at $60{ }^{\circ} \mathrm{C}$ for 10 minutes to avoid undesired hybridization events occurring between partially complementary strands before the incubation with Rad51 protein (Sigma Aldrich) in a 1:1 molar ratio. The reaction occurred in a saline solution containing

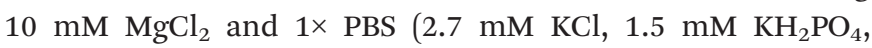
$137.9 \mathrm{mM} \mathrm{NaCl}, 8.1 \mathrm{mM} \mathrm{Na} \mathrm{HPO}_{4} \cdot 7 \mathrm{H}_{2} \mathrm{O}$ ). The solution was incubated in a water bath at $37^{\circ} \mathrm{C}$ for 30 minutes. After the incubation, the mixture was diluted to reach a suitable ssDNA concentration of $1 \mathrm{ng} \mu \mathrm{l}^{-1}$. Afterwards, an $8 \mu \mathrm{l}$ drop of the working solution was deposited on the super-hydrophobic device. The evaporation occurred at standard room temperature and pressure until complete dehydration. The drop-casted samples were directly imaged by TEM without further metallization or preparative steps with a JEOL Jem 1011 electron microscope (JEOL, Japan) equipped with a conventional W-thermoionic electron gun working at $100 \mathrm{kV}$ with a spherical aberration of $5.6 \mathrm{~mm}$. The images were acquired using a Gatan SC-1000 Orius camera equipped with a fiber-optically coupled 11 MP CCD.

\section{Preparation of cortical neurons}

Cortical neurons were obtained from E18.5 C57Bl/6J mouse embryos as previously reported. ${ }^{21}$ Briefly, cortices in ice-cold PBS were digested with $0.125 \%$ trypsin for $30 \mathrm{~min}$ at $37^{\circ} \mathrm{C}$, and mechanically dissociated. Neurons were seeded on poly-Dlysine-coated glass coverslips in Neurobasal medium with $10 \%$ horse serum, $2 \mathrm{mM}$ glutamine and antibiotics (referred to as plating medium). After $3 \mathrm{~h}$, the medium was substituted with Neurobasal supplemented with 2\% B27, 2 mM glutamine and antibiotics (referred to as maintenance medium). All experiments were performed according to the Council of European Communities guidelines (Directive 2010/63/EU of September $22,2010)$ and were approved by the Italian Ministry of Health.

\section{Cell culture and plasma membrane isolation}

Isotonic (0.32 M sucrose, $5 \mathrm{mM}$ Hepes, $1 \mathrm{mM}$ EDTA) and hypotonic ( $5 \mathrm{mM}$ Hepes, $1 \mathrm{mM}$ EDTA) buffer solutions were prepared and adjusted to a pH of 7.4 at $4{ }^{\circ} \mathrm{C} .4 \times 10^{6}$ cells were plated in a $10 \mathrm{~cm}$ Petri dish and cultured for 10 days in main- tenance medium. The culture medium was carefully removed, cells were washed three times with cold $1 \times$ phosphate-buffered saline (PBS) and scraped off by adding $250 \mu \mathrm{l}$ of isotonic buffer. The cell lysate was passed through pipette tips several times until homogeneous. The lysate were centrifuged at $3000 \mathrm{~g}$ for $10 \mathrm{~min}$ at $4{ }^{\circ} \mathrm{C}$ using a tabletop refrigerated centrifuge (\#5417R, Eppendorf s.r.l., Milano, Italy) to pellet the nuclei, whole cells and large cellular debris. The supernatant fraction was diluted 1:10 using the hypotonic buffer solution, homogenized using a teflon Dounce homogenizer (Wheaton, Millville, NJ, USA) and an overhead stirrer RZR 2102 (Heidolph, Schwabach, Germany) (800 rpm, 6 up-and-down strokes), and left on ice for $20 \mathrm{~min}$. After the last centrifugation (Optima ${ }^{\mathrm{TM}}$ MAX ultracentrifuge, Beckman Coulter s.r.l., Milano, Italy) at $200000 \mathrm{~g}$ for $90 \mathrm{~min}$, the supernatant was removed and the membrane pellet was resuspended in $5 \mathrm{mM}$ Hepes, 1 mM EDTA, pH 7.4. Ten $\mu$ l drops were deposited per $\mathrm{cm}^{2}$ of $\mathrm{SH}$ substrate and dried in air overnight at RT.

\section{Characterization of the membranes through imaging}

Transmission electron microscopy. Samples for TEM measurements were fixed in $2 \%$ glutaraldehyde in $0.1 \mathrm{M}$ cacodylate buffer for $1 \mathrm{~h}$. Images were taken on a Jeol JEM 1011 microscope operating at $100 \mathrm{kV}$ acceleration voltage, equipped with a W electron source, a High Contrast Objective Lens and a Gatan Orius CCD camera.

Scanning electron Microscopy. Dried membranes stretched between pillars were fixed as described in ref. 31. Samples were then fixed on SEM stubs with silver glue (Pelco), sputtered with $10 \mathrm{~nm}$ gold/palladium using a Cressington $208 \mathrm{HR}$, and imaged with a Jeol JSM 7500 electron microscope at $5 \mathrm{kV}$, equipped with a cold emission electron source, under low vacuum conditions.

\section{TEM image simulations}

The structural models of the biological systems we have investigated were plotted using UCSF Chimera software, and the corresponding atomic coordinates were extracted using CrystalMaker v 8.7 software. The TEM images were simulated using MacTempas v. 2.3.33 software. Every atomic structure has been subdivided into many phase grating slices; the slices were oriented perpendicular to the propagation of the beam (multislice method). TEM image simulations have been performed using the following electro-optical parameters: $100 \mathrm{keV}$ of acceleration voltage (HT), 1 mrad of convergence angle (Conv), $100 \AA$ of defocus spread (Spread), and $5.6 \mathrm{~mm}$ of spherical aberration coefficient (Cs). For the number of phase grating slices used for every structural model, refer to ESI Table S1.†

\section{Conclusions}

The first advance we show in this paper, through an accurate preparation procedure, is a strong improvement of the image contrast of DNA. In fact, for the first time we could add the 
direct measurements of the backbone and base lengths from the contrast curve.

In the case of DNA-protein interactions, this is, to our knowledge, the first time the resulting complex has been imaged by TEM. We focused on the study of the first interaction stage, i.e. the recognition step. TEM imaging clearly showed the Rad51 docking on the DNA filament and longrange order of the Rad51 oligomer. We plan to study this feature with multiscale molecular dynamics, ${ }^{32}$ in order to further elucidate the stability of this construct and its free energy and compare all parameters in the model with experimental data obtained by HRTEM. We could also propose a docking model based on aspecific binding between $\beta$-sheets and DNA nucleotides and the backbone emerged. This binding also induced a long-range order of protein-protein interactions. We believe that these findings will be important to elucidate further the repair mechanism of Rad51 when ATP will be inserted into the reaction.

Regarding the imaging of the suspended membranes, we showed that this method permits the study of a free-standing membrane suspension in which a single membrane layer can be imaged at low dose, good contrast and few- $\AA$ resolution that allows resolving and characterizing individual ion channels and lipid bilayer metrology. Moreover, the amount of information present in the field of view of HRTEM images is compatible for a further "single particle" analysis for protein structural studies as in cryo-EM. The clear advantage of the present approach is that it allows background-free TEM imaging of complex samples that have been prepared under native physiological conditions at an unprecedented structural resolution level.

The use of the state-of-the-art TEMs, equipped with a spherical aberration corrector of the lens system, and the most advanced camera devices ${ }^{33,34}$ will further enable the present method to reach imaging details close to Ångstrom resolution for biological samples as in solid state samples, where low contrast and dose damage do not burden the image formation.

\section{Author contributions}

E. D. F. conceived the whole project, participated in all phases: super-hydrophobic device conceiving, DNA structure solution, TEM imaging, data analysis and writing the manuscript; Monica M. participated in conceiving and DNA and DNA/ protein complex preparation, performed experiments, participated in TEM imaging and data analysis, and contributed to manuscript writing; T. L. participated in conceiving and membrane preparation, performed experiments, participated in TEM imaging and data analysis, and contributed to manuscript writing; A. F. did TEM measurements and TEM simulation and contributed to manuscript writing; Alessandro G. did TEM image simulation and contributed to manuscript writing; Manola M. participated in preparing the solutions and DNA suspension; M. A. realized the super-hydrophobic devices; S. L. performed TEM measurements and imaging optimization; L. T. participated in suspension optimization and preparing the solutions; G. D. participated in preparation and optimization of the sample and its purification through Raman scattering; B. T. and Andrea G. participated in preparing the samples and optimizing their drying on a super-hydrophobic substrate; F. C and F. B. participated in the membrane sample supply and preparation and in manuscript writing and corrections. All the authors discussed and commented on the manuscript.

\section{Competing financial interests}

The authors declare no competing financial interests.

\section{Acknowledgements}

The authors acknowledge financial support from the KAUST start-up funding and from the Italian Ministry of Health under project no. GR-2010-2320665 and GR-2010-2311677.

\section{References}

1 M. Marini, A. Falqui, M. Moretti, T. Limongi, M. Allione, A. Genovese, S. Lopatin, L. Tirinato, G. Das, B. Torre, A. Giugni, F. Gentile, P. Candeloro and E. Di Fabrizio, Sci. $A d v ., 2015,1, \mathrm{e} 1500734$.

2 R. F. Egerton, P. Li and M. Malac, in Micron, 2004, vol. 35, pp. 399-409.

3 K. N. Goldie, P. Abeyrathne, F. Kebbel, M. Chami, P. Ringler and H. Stahlberg, Methods Mol. Biol., 2014, 1117, 325-341.

4 W. Kuhlbrandt, eLife, 2014, 3, e03678.

5 Y. Lu, C. Peng, Y. Ganesan, J. Y. Huang and J. Lou, Nanotechnology, 2011, 22, 6.

6 F. Gentile, M. Moretti, T. Limongi, A. Falqui, G. Bertoni, A. Scarpellini, S. Santoriello, L. Maragliano, R. Proietti Zaccaria and E. Di Fabrizio, Nano Lett., 2012, 12, 64536458.

7 W. Ranatunga, D. Jackson, R. A. Flowers and G. E. O. Borgstahl, Biochemistry, 2001, 40, 8557-8562.

8 B. J. G. E. Pieters, M. B. van Eldijk, R. J. M. Nolte and J. Mecinović, Chem. Soc. Rev., 2015, 24-39.

9 R. M. Story, I. T. Weber and T. A. Steitz, Nature, 1992, 355, 318-325.

10 H. Aihara, Y. Ito, H. Kurumizaka, S. Yokoyama and T. Shibata, J. Mol. Biol., 1999, 290, 495-504.

11 L. Pellegrini, D. S. Yu, T. Lo, S. Anand, M. Lee, T. L. Blundell and A. R. Venkitaraman, Nature, 2002, 420, 287-293.

12 D. S. Shin, L. Pellegrini, D. S. Daniels, B. Yelent, L. Craig, D. Bates, D. S. Yu, M. K. Shivji, C. Hitomi, A. S. Arvai, N. Volkmann, H. Tsuruta, T. L. Blundell, A. R. Venkitaraman and J. A. Tainer, EMBO J., 2003, 22, 4566-4576. 
13 L. T. Chen, T. P. Ko, Y. C. Chang, K. A. Lin, C. S. Chang, A. H. J. Wang and T. F. Wang, Nucleic Acids Res., 2007, 35, 1787-1801.

14 D. S. Shin, L. Pellegrini, D. S. Daniels, B. Yelent, L. Craig, D. Bates, D. S. Yu, M. K. Shivji, C. Hitomi, A. S. Arvai, N. Volkmann, H. Tsuruta, T. L. Blundell, A. R. Venkitaraman and J. A. Tainer, EMBO J., 2003, 22, 4566-4576.

15 O. R. Davies and L. Pellegrini, Nat. Struct. Mol. Biol., 2007, 14, 475-483.

16 L. Krejci, J. Damborsky, B. Thomsen, M. Duno and C. Bendixen, Mol. Cell. Biol., 2001, 21, 966-976.

17 S. L. Brenner, A. Zlotnick and J. D. Griffith, J. Mol. Biol., 1988, 204, 959-972.

18 J. Heuser and J. Griffith, J. Mol. Biol., 1989, 210, 473-484.

19 F. E. Benson, A. Stasiak and S. C. West, EMBO J., 1994, 13, 5764-5771.

20 R. Rohs, S. M. West, A. Sosinsky, P. Liu, R. S. Mann and B. Honig, Nature, 2009, 461, 1248-1253.

21 F. Cesca, A. Yabe, B. Spencer-Dene, J. Scholz-Starke, L. Medrihan, C. H. Maden, H. Gerhardt, I. R. Orriss, P. Baldelli, M. Al-Qatari, M. Koltzenburg, R. H. Adams, F. Benfenati and G. Schiavo, Cell Death Differ., 2012, 19, 194-208.

22 X. Tao, A. Lee, W. Limapichat, D. A. Dougherty and R. MacKinnon, Science, 2010, 328, 67-73.

23 B. C. Bennett, M. D. Purdy, K. A. Baker, C. Acharya, W. E. McIntire, R. C. Stevens, Q. Zhang, A. L. Harris, R. Abagyan and M. Yeager, Nat. Commun., 2016, 7, 8770.
24 P. S. Miller and A. R. Aricescu, Nature, 2014, 512, 270275.

25 C.-S. Chiu, K. Jensen, I. Sokolova, D. Wang, M. Li, P. Deshpande, N. Davidson, I. Mody, M. W. Quick, S. R. Quake and H. a. Lester, J. Neurosci., 2002, 22, 1025110266.

26 M. V. Jones and G. L. Westbrook, Neuron, 1995, 15, 181191.

27 M. V. Jones, Y. Sahara, J. a. Dzubay and G. L. Westbrook, J. Neurosci., 1998, 18, 8590-8604.

28 A. Bartesaghi, A. Merk, S. Banerjee, D. Matthies, X. Wu, J. L. S. Milne and S. Subramaniam, Science, 2015, 348, 1147-1151.

29 X. Li, P. Mooney, S. Zheng, C. R. Booth, M. B. Braunfeld, S. Gubbens, D. A. Agard and Y. Cheng, Nat. Methods, 2013, 10, 584-590.

30 Y. Fujiyoshi and N. Uyeda, Ultramicroscopy, 1981, 7, 189192.

31 T. Limongi, F. Cesca, F. Gentile, R. Marotta, R. Ruffilli, A. Barberis, M. Dal Maschio, E. M. Petrini, S. Santoriello, F. Benfenati and E. Di Fabrizio, Small, 2013, 9, 402-412.

32 S. Pricl, M. Ferrone, M. Fermeglia, F. Amato, C. Cosentino, M. M. C. Cheng, R. Walczak and M. Ferrari, Biomed. Microdevices, 2006, 8, 277-290.

33 C. Ophus, J. Ciston, J. Pierce, T. R. Harvey, J. Chess, B. J. McMorran, C. Czarnik, H. H. Rose and P. Ercius, Nat. Commun., 2016, 7, 10719.

34 D. Van Dyck and F.-R. Chen, Nature, 2012, 486, 243-246. 